\title{
Unique parameter identification of a cardiovascular system model using feedback control
}

\author{
Christopher E. Hann, J. Geoffrey Chase, Thomas Desaive, Claire Froissart, James Revie, David \\ Stevenson and Geoffrey M. Shaw
}

\begin{abstract}
Lumped parameter differential equation models are a common approach to modeling the cardiovascular system. However, there are highly non-linear valve dynamics inherent in these models which makes parameter identification difficult. Standard methods for parameter identification rely on gradient descent, which can often converge to wrong solutions, particularly as the number of parameters increases. This paper presents a new concept of parameter identification, applied to a 2 chamber model of the left ventricle systemic system. The changes in the parameters are treated as an actuation force into a feed back control system, where the reference output is taken to be steady state values of measured volume and pressure. The major advantage of the method is that when it converges, it must be at the global minimum, so that the correct solution is always found. The method is validated in both simulation and on a porcine model of pulmonary embolism. Very accurate matches to clinically measured left ventricle volume/pressure and aortic pressure waveforms are achieved, and the method gives considerable flexibility in capturing any required geometrical feature in the waveforms.
\end{abstract}

\section{INTRODUCTION}

$\mathrm{C}$ ARDIOVASCULAR system (CVS) models are inherently non-linear due to the pulsatile nature of the heart. There are also typically many parameters required to describe the CVS in detail [1-5]. Thus typically, only small subsets of the parameter set can be identified (e.g. [3]). As a result, a majority of the parameter set has to be fixed at population values, which demands prior knowledge on the state of patients and a homogeneity between patients that may not exist.

In critical care, a patient's condition can change rapidly and therefore any pre-assumption on parameters may jeopardise accurate diagnosis. Furthermore, the more complex a model becomes, the larger the set of unidentifiable parameters, and the greater the number of dynamics that may differ from the actual. Increased computational requirements with increasing parameters or

Manuscript received April 14, 2009.

C. E. Hann, J. G. Chase, J. Revie and D. Stevenson are with the Department of Mechanical Engineering, University of Canterbury, Christchurch, New Zealand (e-mail: Chris.Hann@canterbury.ac.nz, Geoff.Chase@canterbury.ac.nz).

T. Desaive is with the Institute of Physics, University of Liege, Belgium (e-mail: tdesaive@ulg.ac.be)

C. F. Froissart is with the Universite de Technologie de BelfortMontbeliard, France (e-mail: claire.froissart@gmail.com)

G. M. Shaw is with the Department of Intensive Care, Christchurch Hospital (e-mail: geoff.shaw@cdhb.govt.nz) model complexity will also limit real-time patient specific application at the bedside.

This research takes a different approach, by first developing simplified, fully identifiable, patient specific models, that are based around the clinical data available in an Intensive Care Unit. These models can serve as a bridge to identify more complicated and physiologically accurate models as required to predict the observed patient hemodynamic responses. In the simplified models, patient specific dynamics are only considered if they can be uniquely identified from the given data. Due to the simplified structure of these models, it is then possible to analyze individual geometric effects of given input parameters on the output. This information will lead to the minimal set of features in the outputs, that are required for adequate cardiac diagnosis.

The model analyzed in this paper is a simplified model of the left-ventricle systemic system, but the results could be readily extended to any cardiovascular system model. The model is shown to be uniquely identifiable given specific features that a readily available in an ICU. For a given set of data, the unique parameters are found by an iterative approach, where they are updated depending on ratios of the maximum and minimum volumes and pressures in the measured data. This approach is very similar to proportional feed back control, except rather than taking the difference between the reference and measured output, the ratio is used.

The results are tested in simulation with random noise added and on some measured data from a pulmonary embolism experiment on pigs.

\section{METHODOLOGY}

\section{A. Cardiac model}

The cardiac model, is essentially a sub-model of a previously developed six chamber cardiac model [4-5] with inertial effects and ventricular interaction. A schematic of the simplified model is shown in Figure 1. Note that this model can easily be extended to the right ventricle as well, but for this paper only the left side is considered. In Figure 1 the vena cava and pulmonary vein pressures are assumed constant and the model can be expressed in terms of differential equations defined: 


$$
\begin{gathered}
\dot{V}_{l v}=H\left(Q_{m t}\right) Q_{m t}-H\left(Q_{a v}\right) Q_{a v} \\
\dot{P}_{a o}=E_{a o} H\left(Q_{a v}\right) Q_{a v}-E_{a o}\left(\frac{P_{a o}-P_{v c}}{R_{s y s}}\right) \\
Q_{m t}=\frac{P_{p v}-P_{l v}}{R_{m t}} \\
Q_{a v}=\frac{P_{l v}-P_{a o}}{R_{a v}} \\
P_{l v}=e(t) E_{e s, l v f} V_{l v}+P_{t h} \\
e_{n e w}(t)=\frac{P_{l v, f u l l}-P_{t h}}{V_{l v, f u l l}}
\end{gathered}
$$

The parameters $P_{l v, \text { full }}$ and $V_{l v, \text { full }}$ in Equation (6) are defined to be either the full model outputs of [4] from a healthy human parameter set with a heart beat period of 0.75 seconds or from measured data. Hence Equation (6) represents a population driver function, which could be scaled to represent different heart beats. However, the shape could be altered as required to capture individual patient response.

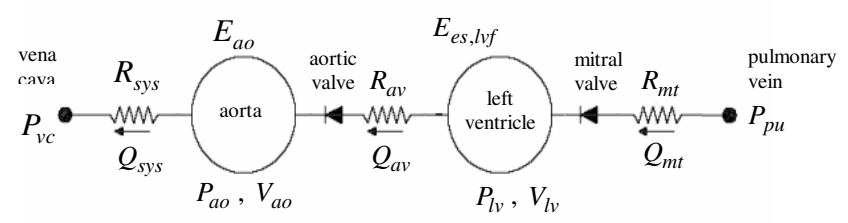

Fig. 1: The left ventricle-systemic system simplified model

\section{B. Unique parameter identification}

The unknown patient specific parameters, denoted $X$, that are optimized for the left ventricle model of Equations (1)(6) are defined:

$$
X \equiv\left\{P_{p v}, E_{e s, l v f}, E_{a o}, R_{m t}, R_{a v}, R_{s y s}\right\}
$$

The parameter $P_{v c}$ in Equation (2) is assumed known, since it would be found from either identifying the right ventricle system, or by direct measurement of the central venous pressure, which is common in an intensive care unit.

There are 6 unknown parameters in Equation (7) to be identified in the model of Figure 2 (a). Therefore, the measured maximum/minimum left ventricle volume and aortic pressure can only uniquely identify 4 of these parameters. However, the timing of the mitral valve closure corresponds to the end of the atrial contraction which can be detected by the end of the $\mathrm{P}$ wave on an electrocardiogram (ECG) [6]. Alternatively, since the left and right atriums contract close to simultaneously, the mitral valve closure can also be calculated from the "a wave" in the central venous pressure waveform [7]. The central venous pressure is commonly measured in the ICU.

These observations demonstrate an important concept, which is to utilize features from physiological waveforms to improve identifiability without having to explicitly model the effects. The pressure in the pulmonary vein $P_{p v}$ or the filling pressure of the simplified model of Figure 1 corresponds to the left ventricle pressure at the mitral valve closure. Hence, $P_{p v}$ can be estimated by the formula:

$$
P_{p v}=P_{l v}\left(t_{d 2}\right), t_{d 2} \equiv \text { time of mitral valve closure }
$$

A further important feature available is the maximum gradient or inflection point in the ascending aortic pressure wave. The parameter which has a significant effect on the maximum aortic pressure gradient is the resistance in the aortic valve $R_{a v}$. Define:

$$
\alpha\left(R_{a v}\right)=\frac{P_{a o, \text { true }}\left(t_{\text {inflect }}\right)-P_{a o, \text { true }}\left(t_{\text {min }}\right)}{P_{a o, a p p r o x}\left(t_{\text {inflect }}\right)-P_{a o, a p p r o x}\left(t_{\text {min }}\right)}
$$

where $P_{a o, a p p r o x}$ and $P_{a o, t r u e}$ are the simulated and "measured" aortic pressures, $t_{\min }$ is the time of minimum aortic pressure and $t_{\text {inflect }}$ is the time of maximum aortic pressure gradient. Equation (9) is an approximation to the ratio of the maximum gradients of $P_{a o, a p p r o x}$ to $P_{a o, t r u e}$ and is used to avoid having to differentiate the aortic pressure which may be noisy. Simulation has shown that the variable $\alpha$ in Equation (9), changes inversely proportional to $R_{a v}$ with all other parameters held at their nominal values. Specifically, if $R_{a v}$ increases by a factor of 2, with all other parameters fixed, $\alpha$ approximately reduces by a factor of 2 , with a order of magnitude less effect on the maximum and minimum volumes/pressures. This result motivates an approximation to $R_{a v}$ :

$$
R_{a v, \text { approx }}=\frac{P_{a o, a p p r o x}\left(t_{\text {inflect }}\right)-P_{a o, a p p r o x}\left(t_{\min }\right)}{P_{a o, \text { true }}\left(t_{\text {inflect }}\right)-P_{a o, \text { true }}\left(t_{\min }\right)} R_{a v, \text { old }}
$$

However, for Equation (8) and (10) to be valid approximations, to $P_{p v}$ and $R_{a v}$, the approximations $P_{l v, a p p r o x}$ and $P_{a o, a p p r o x}$ need to be as accurate as possible. The solution proposed, is to first ensure that the maximum/minimum simulated volumes and aortic pressures are precisely matched to the measured values for given initial (but essentially arbitrary) estimates of $P_{p v}$ and $R_{a v}$. At the end of this optimization, $P_{p v}$ and $R_{a v}$ are updated using Equations (8) and (10).

Simulation has shown that increasing the parameters $E_{e s, l v}$, and $R_{m t}$ separately by factors of 2 decrease the mean volume, and stroke volume by factors close to 2 . On the other hand, increasing the parameters for $E_{a o}$ and $R_{s y s}$ proportionally increase the pulse pressure difference and the mean aortic pressure. These results motivate the following definitions: 


$$
\begin{aligned}
& E_{e s, l v f, \text { approx }}=\left(\frac{V_{l v, \text { min,approx }}+V_{l v, \text { max }, \text { approx }}}{V_{l v, \text { min,true }}+V_{l v, \text { max }, \text { true }}}\right) E_{e s, l v f, \text { old }} \\
& R_{m t, \text { approx }}=\left(\frac{S V_{\text {approx }}}{S V_{\text {true }}}\right) R_{m t, \text { old }} \\
& E_{\text {ao,approx }}=\left(\frac{P P_{\text {true }}}{P P_{\text {approx }}}\right) E_{\text {ao,old }} \\
& R_{s y s, a p p r o x}=\left(\frac{P_{a o, \text { max }, \text { true }}+P_{a o, \text { min }, \text { true }}}{P_{a o, \text { max }, \text { approx }}+P_{a o, \text { min }, \text { approx }}}\right) R_{s y s, \text { old }}
\end{aligned}
$$

The tests for the parameter identification method are done first in simulation with noise, and then on animal data. In the tests with noise, the "measured data" is taken to be the output of the six chamber model of Figure 1. For the animal experiments, the measured data is from catheters [8]. In both cases:

data $\equiv$ mean $V_{l v}, S V, P P$, mean $P_{a o}, P_{a o}(t), t_{d 2}$

where:

$$
\begin{aligned}
& \text { mean } V_{l v}=\frac{V_{l v, \text { max }}+V_{l v, \text { min }}}{2}, S V=V_{l v, \text { max }}-V_{l v, \text { min }}, \\
& P P=P_{a o, \max }-P_{a o, \min }, \text { mean } P_{a o}=\frac{P_{a o, \max }+P_{a o, \min }}{2}, \\
& t_{d 2} \equiv \text { end diastolic time }
\end{aligned}
$$

The overall parameter identification method is summarized in Figure 2.

Step1 Choose arbitrary set of input parameters including $P_{p u}$ and $R_{a v}$

Step2 Simulate model of Equations (1)-(6)

$\underline{\text { Step3 }}$ Compute approximations to $E_{e s, l v f}, R_{m}, E_{a o}$ and $R_{s y s}$ from Equations (11)-(14).

Step4 Simulate model Equations (1)-(6).

$\underline{\text { Step5 }}$ If the maximum volumes and aortic pressures are matched within a given tolerance go to Step 6, otherwise go back to Step 3.

Step6 Compute $P_{v u}$ and $R_{a v}$ from Equations (8) and (10). If they have changed by less than $1 \%$ go to Step 7 otherwise go back to Step 3 .

$\underline{\text { Step7 }}$ Output final solution and identified parameters

Fig. 2: Parameter identification algorithm for Figure 1

Notice that the updates in Equations (10)-(14), are similar to proportional feedback control. That is, these parameters can be considered to be the actuation force that controls the reference output, which is the data in Equation (15). The only difference is that instead of applying a force proportional to the difference between the reference and actual output, the force is proportional to the ratio of the reference to actual output.

\section{RESULTS AND DISCUSSION}

This section first validates the simplified modelling approach of Figure 1 and the parameter identification method of Figure 2, against simulated data from the full six chamber cardiac model [3,4]. Measurement noise is simulated by corrupting the simulated data with random uniformly distributed noise. The noise is defined:

$$
\text { uniform noise } \equiv 5 \% \text { in } P_{a o}(t), 10 \% \text { in SV, } 10 \% \text { in } t_{d 2}
$$

where for the end-diastolic filling time $t_{d 2}$ in Equation (15), the $10 \%$ noise is relative to the length of time of the diastole. The noise is made less for the pressure, since it is assumed that a catheter measures the aortic pressure waveform, which is standard in an ICU. Modelling error is also present in the simplified model of Figure 1 with respect to the full six chamber cardiac model [4,5].

The model and methods of the left ventricle-systemic system are then tested on clinical data from a pulmonary embolism animal model experiment [8].

\section{A. Convergence of algorithm and effectiveness of modeling approach}

To demonstrate the fast and accurate convergence of the algorithm of Figure 3, and to assess the suitability of the simplified models of Figure 2 in describing the full model of Figure 1, a healthy human is considered [4]. In this case no noise is added so an accurate characterization of the accuracy of the simplified models can be obtained. The assumed measured data is:

$$
\text { data } \equiv \text { mean } V_{l v}, S V, P P \text {, mean } P_{a o}, P_{a o}(t), t_{d 2}
$$

where $P_{a o}$ is given as a function of time since it is continuously measured.

The left ventricle volume matches very closely to the true volume with maximum errors of $1.6 \%$ and $2.6 \%$ during filling and ejection, and errors of $0.00016 \%$ and $0.00014 \%$ in the maximum and minimum volumes respectively. Similarly, the identified aortic pressure closely captures the true pressure with maximum errors of $1.8 \%$ and $0.2 \%$ during ejection and filling, and errors of $0.0026 \%$ and $0.0015 \%$ in the maximum and minimum aortic pressures respectively. The error in the maximum ventricle pressure is $2.1 \%$. 
For the identified parameters, the largest error is $21.7 \%$ in $R_{a v}$ but this is largely due to the already small value. There is also an error of $8.1 \%$ in $E_{e s, l v f}$ which reflects the modelling error of Figure 1 with respect to the six chamber model of [4]. These results show that the simplified model of Equations (1)-(6) is a very accurate representation of the full model, with small errors due to modelling error.

The accurate results also provide an initial validation of the parameter identification method of Figure 2, with very fast convergence obtained, even when starting significantly far away from the solution. Figure 3 plots the maximum volume and Figure 4 plots $R_{a v}$ against the number of iterations, for an initial guess containing $300-400 \%$ error in all the parameters. One iteration is equivalent to one numerical simulation of Equations (1)-(6) that occurs in step 3 of Figure 2. The maximum volume converges in about 10 iterations, and remains largely unaffected by changes in $R_{a v}$, where $R_{a v}$ takes about 24 iterations to converge within $1 \%$. The convergence time of $R_{a v}$ can be reduced by a factor of 2 by re-defining $R_{a v}$ in Equation (10) by:

$$
R_{a v, \text { approx }}=\left(\frac{P_{a o, \text { approx }}\left(t_{\text {inflect }}\right)-P_{a o, \text { approx }}\left(t_{\text {min }}\right)}{P_{a o, \text { true }}\left(t_{\text {inflect }}\right)-P_{a o, \text { true }}\left(t_{\text {min }}\right)}\right)^{\text {gain }} R_{a v, \text { old }}
$$

and setting a gain of 3, as shown in Figure 4. Importantly, once the method converges, this implies that the ratios in Equations (11)-(14) must be unity, otherwise the parameters would keep changing. Hence, the method can never reach a local minima and must always stop at the global minimum.

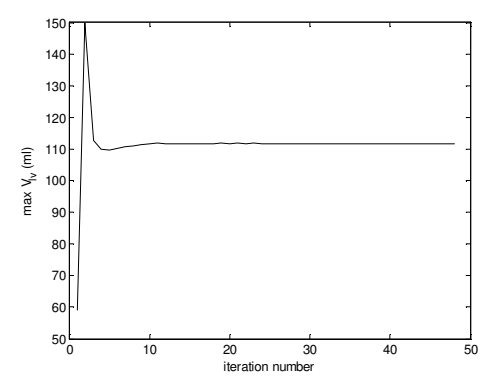

Fig. 3: Convergence of the maximum volume using algorithm of Figure 3.

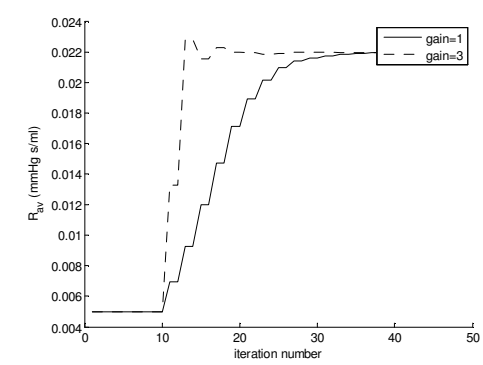

Fig. 4: Convergence of Rav in the algorithm of Figure 3 using two gains of 1 and 3 in Equation (41).
In summary the parameter identification method of Figure 3 is very fast and accurate independent of starting point, and the simplified model of Figure 1 closely capture the full model dynamics of [4]. Importantly, the data of Equation (15) is sufficient to uniquely identify all six parameters in Equation (7). The simpler nature of Figure 1 also means the model simulations are dramatically faster.

\section{B. Validation on an animal model and clinical implementation}

To demonstrate the clinical potential for the methods developed, data from a porcine pulmonary embolism experiment is used. The data is obtained from the Hemodynamics Research Laboratory, University of Liege, Belgium. In the experiments, a pig is injected with autologous blood clots every two hours to simulate pulmonary embolism [8].

As a simple proof of concept, the left ventricle model of Figure 1(a) and the method of Figure 2 are applied using measured waveforms for one of the pigs at two time points of 30 minutes and 210 minutes. No ECG or the central venous pressure waveform was available, therefore, the end-diastolic filling time $t_{d 2}$ in Equation (15) was manually estimated from the left ventricle volume profile. A driver function is derived in a similar way to Equation (6), but with $P_{l v, \text { full }}$ and $V_{l v, \text { full }}$ replaced by the measured left ventricle pressure and volume, and $P_{t h}$ is set to 0 , since the pig is open chest. The resulting function is smoothed by least squares cubic splines and normalized so the maximum point is 1 and the time interval of one heart beat is the healthy value of 0.75 . To account for different heart rates, the generic shape is defined:

$$
\hat{e}(t)=\bar{e}\left(\frac{0.75}{\text { period }} t\right)
$$

where $\bar{e}(t)$ is experimentally derived from the healthy state of several pigs based on an average response and is shown in Figure 7.

To account for individual pig variations the final driver function is defined:

$$
\begin{aligned}
e(t) & =\hat{e}(\alpha t+\beta), \quad 0<t<\text { period } \\
\alpha & =\frac{t_{\nabla a o, \text { min }}-t_{a o, \text { min }}}{t_{\text {inflect }, 2}-t_{\text {inflect }, 1}}, \beta=\frac{t_{a o, \text { min }} t_{\text {inflect }, 2}-t_{\nabla a o, \text { min }} t_{\text {inflect }, 1}}{t_{\text {inflect }, 2}-t_{\text {inflect }, 1}}
\end{aligned}
$$

where $t_{a o, \min }$ is the time of minimum aortic pressure, $t_{\nabla a a_{\text {, min }}}$ is the time of the minimum (or maximum negative) 
aortic pressure gradient, and $t_{\text {inflect }, 1}$ and $t_{\text {inflect }, 2}$ are the first and second inflection points of $\hat{e}$ in Equation (20).

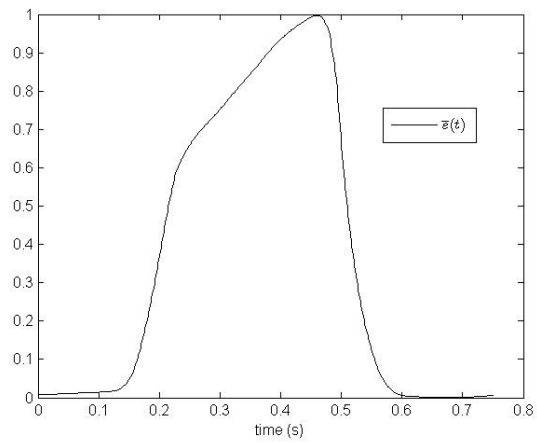

Fig. 5: Experimentally derived driver function based on Equation (20)

Specifically, $t_{\nabla a o, \min }$ is well known to correspond to the minimum left ventricle pressure gradient (or inflection point) which always occurs just before the dicrotic notch, and thus corresponds to the aortic valve closure. The volume is approximately constant at this point, and therefore, the formula of Equation (6) shows that $t_{\text {inflect }, 2}$ should be equal to $t_{\nabla \text { ao,min }}$. The maximum left ventricle pressure gradient, is also known to occur just before the aortic valve opens, which corresponds closely to $t_{a o, \min }$. Therefore since the volume is constant at this point as well, $t_{\text {inflect }, 1}$ should be equal to $t_{a o, \min }$. The time scaling transformation in Equation (21) ensures that the inflection points of the driver function correspond to $t_{a o, \min }$ and $t_{\nabla a o, \min }$ as required. Equations (20) and (21) therefore provide a way of approximately identifying a patient (pig) specific driver function. Further clinical experiments and trials on humans are needed to classify to what degree of accuracy the driver function is required to be for adequate cardiac diagnosis.

Figure 6(a) shows the result of applying the algorithm of Figure 2 on the one of the pigs at 30 minutes into the pulmonary embolism experiment. This figure is compared to a special case of a fixed $\mathrm{Ppv}=2$ and $\mathrm{Rav}=0.46$ in Figure 6(b). In both cases the maximum and minimum values of Vlv and Pao (not shown) are accurately captured, but there are errors of $34 \%$ and $84 \%$ in the parameters Ees,lvf and Rmt. The errors in Eao and Rsys are less than 5\%. However, the parameter Ees,lvf appears relatively robust and is virtually unaffected by changes in Ppu. For example if $\operatorname{Rav}<0.2$, the errors of Ees,lvf are less than 10\%. But these results highlight the importance of the data set in Equation (15) to accurately identify the model as well as finding a unique parameter set.
The PV curve and aortic pressure waveform corresponding to the correct parameters in Figure 6(a) are plotted in Figure 7, showing a close match. Notice how the first third to a half of the ascending aortic pressure is matched almost exactly. The high degree of accuracy in this period is due to the parameter identification method forcing the inflection point of the model to match the inflection point of the data. This result further shows the power of the method of Figure 2 as any feature that the model of Figure 1 is capable of matching, can be precisely captured independent of starting point, with very fast convergence and very minimal computation.
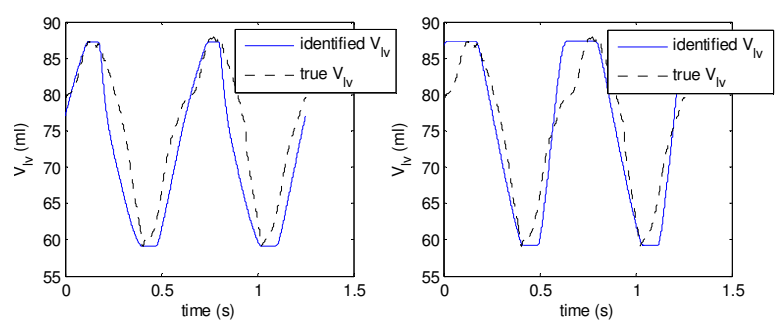

Fig. 6: Applying the algorithm of Figure 2 to the pig data. (a) identifying all parameters (b) fixing $P_{p u}$ and $R_{a v}$
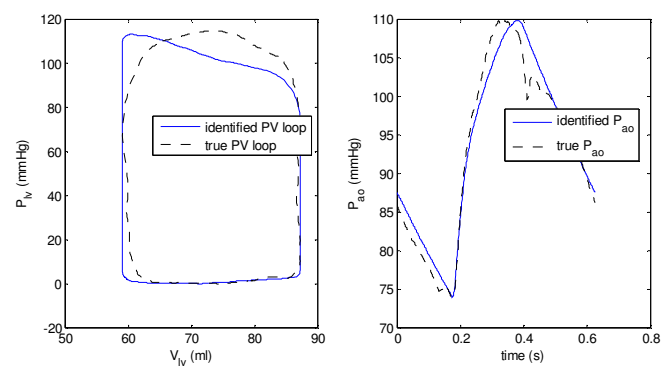

Fig 7: Identification results using correct parameters of Fig. 6(a). (a) Pressure-volume curve (b) Aortic pressure waveform

It has been shown that the ascending aortic waveform inflection point is a predictive factor for all-cause and cardiovascular mortality in patients with chronic renal failure on hemodialysis [9]. Many other studies have also shown features in the continuous aortic pressure waveform to help diagnose disease states and to monitor improvements due to therapy. Therefore, this modelling and parameter identification approach has the potential to aggregate key clinical information and any significant correlations between parameters observed in the literature. The goal is to build on the concepts in this paper and prior work, to create one unified and uniquely identifiable physiological model for better managing patient specific cardiovascular response in critical care.

\section{Summary}


Three major concepts were introduced in this paper.

- A simplified fully identifiable sub-model that closely captures a six chamber model with ventricular interaction, pericardium and inertial effects, and are capable of uniquely characterizing measured clinical response of pig data

- A parameter identification method based on a proportional feedback control system. The formulation allows any chosen geometrical feature to be precisely captured with very minimal computation, for example the end-diastolic filling time and maximum ascending aortic gradient. Once the method converges, by definition of the control law, the global minimum must be reached. Therefore local minima which commonly occur in standard non-linear regression are avoided.

- Results in both simulation and clinical data suggest the maximum and minimum volumes are not needed for disease state diagnosis, with the addition of enddiastolic filling time and continuous ascending aortic pressure.

\section{CONCLUSION}

Two simplified models of the left ventricle systemic and right ventricle pulmonary systems were developed that closely matched output data from a full cardiovascular system model with pericardium and ventricular interaction dynamics. The left ventricle system model was tested in simulation with up to $10 \%$ random uniformly distributed noise added to the data. The model was shown to be uniquely identifiable with the addition of the end-diastolic filling time and continuous information from the aortic pressure waveform. Furthermore, the extra data used, that is readily available in an ICU, enabled the mean volume to be added as an extra unknown parameter with minimal effect on identifiability. This result has significant potential clinically, as the mean or maximum/minimum volumes are much harder to measure, where the stroke volume is relatively easy and more common.

The approach of breaking down the six chamber heart model into separate uniquely identifiable models is general and could be applied to any complex lumped parameter CVS model. In particular, future work will utilize the simpler models to allow rapid identification of the 8 chamber model [10] and any other added dynamics as required to diagnose cardiac disease states and characterize therapy response.

The clinical and simulated results both suggest that potentially better model-based diagnostic capability could be obtained with the addition of continuous aortic pressure information, and either ECG or the central venous pressure waveform to obtain the end-diastolic time. This enhanced capability has been shown to be not significantly reduced when removing volume $V_{l v, \text { max }} / V_{l v, \text { min }}$ measurements. The results thus show the potential for practical implementation of a model-based cardiac diagnosis/therapeutics system in the ICU based on readily measurable parameters.

\section{REFERENCES}

[1] Chung D. C., S. C. Niranjan, J. W. Clark Jr, A. Bidani, W. E. Johnston, J. B. Zwischenberger, and D. L. Traber, "A dynamic model of ventricular interaction and pericardial influence," Am. J. Physiol., vol. 272, pp. H2942-2962, 1997.

[2] Luo C., D.L. Ware, J.B. Zwischenberger, and J. W. C. Jr, "Using a human cardiopulmonary model to study and predict normal and diseased ventricular mechanics, septal interaction, and atrioventricular blood flow patterns," J. Cardiovasc. Eng., vol. 7, pp. 1731, 2007.

[3] Mukkamala R. and R. J. Cohen, "A forward model-based validation of cardiovascular system identification," Am. J. Physiol. Heart Circ. Physiol., vol. 281, pp. H2714-H2730, 2001.

[4] Hann C. E., J. G. Chase, and G. M. Shaw, "Efficient implementation of non-linear valve law and ventricular interaction dynamics in the minimal cardiac model," Comput Methods Programs Biomed, vol. 80(1), pp. 65--74, Oct 2005.

[5] Smith B. W., J. G. Chase, R. I. Nokes, G. M. Shaw, and G. Wake, "Minimal haemodynamic system model including ventricular interaction and valve dynamics," Medical Engineering \& Physics, vol. 26(2), pp. 131-139, 2004.

[6] Guyton A. C. and J. E. Hall, Textbook of medical physiology, 10th ed.: W.B. Saunders Company, Philadelphia, 2000.

[7] Muralidhar K., "Central venous pressure and pulmonary capillary wedge pressure monitoring," Indian J. Anaesth, vol. 46, pp. 298-303, 2002.

[8] Ghuysen A., B. Lambermont, P. Kolh, V. Tchana-Sato, D. Magis, P. Gerard, M. Mommens, N. Janssen, T. Desaive, and V. D'Orio, "Alteration of Right Ventricular-Pulmonary Vascular Coupling in a Porcine Model of Progressive Pressure Overloading," Shock, vol. 29(2), pp. 197-204, 2007.

[9] Ueda H., T. Hayashi, K. Tsumura, K. Yoshimaru, Y. Nakayama, and J. Yoshikawa, "Inflection point of ascending aortic waveform is a predictive factor for all-cause and cardiovascular mortality in patients with chronic renal failure on hemodialysis," Am J Hypertens, vol. 17, pp. 1151-5, 2004.

[10] Starfinger C., J. G. Chase, C. E. Hann, G. M. Shaw, B. Lambermont, A. Ghuysen, P. Kolh, P. Dauby, and T. Desaive, "Model-based identification and diagnosis of a porcine model of induced endotoxic shock with hemofiltration," Math Biosci, vol. 216(2), pp. 132-139, 2008 . 